\title{
STUDY ON MODELING AND CONTROL OF EXCAVATOR
}

\author{
$\underline{\text { Le Quang Hoan }}{ }^{1}$, Chan Se Jeong ${ }^{1}$, Hack Sun Kim ${ }^{1}$, He Lim Yang ${ }^{1}$, and Soon Yong Yang ${ }^{2} *$ \\ ${ }^{1}$ Graduate School of Mechanical and Automotive Engineering, University of Ulsan, Ulsan, Korea \\ ${ }^{2}$ Department of Mechanical and Automotive Engineering, University of Ulsan, Ulsan, Korea \\ *Corresponding author(soonyy@ulsan.ac.kr)
}

\begin{abstract}
An excavator is a typical hydraulic heavy-duty human-operated machine used in general versatile construction operations, such as digging, carrying loads, grounding and dumping loads. However, operating excavators in the hazard environment such as earthquake, nuclear decomposition etc is not suitable for human to operate on site. Therefore, automation in excavator has been investigated to protect the operators from the harmful environments. To find a feasible way to control excavator, firstly the Full Kinematic and dynamic model of the excavator's manipulator which three degrees of freedom were studied. The inverse kinematic equations are used to determine the joint angles and the actuator lengths corresponding to a specific position and orientation of the bucket, given in the base coordinate system. To control the excavator's manipulator, the computed torque control are using and some simulation results are given.
\end{abstract}

Keywords: Excavator, Modeling, Automation, Control, Manipulator

\section{INTRODUCTION}

Excavation is an importance work in mining, earth removal and general earthworks. Excavation productivity (amount of work done), efficiency (cost of work done in terms of labor and machinery) and operator safety, particularly in underground mining or during the removal of hazardous waste, are constantly under pressure from industry. After decades of increases in machine size and power, practical limits are now being approached and automation is being sought for further improvements. Furthermore, computing and sensing technologies have reached to a stage where they can be applied to automatic excavation. Nowadays, automation in excavators has been studied by several researchers and so on. Among the fundamental element used as a basis for developing complicated tasks, the Control of excavator's manipulator is an important and basic task in autonomous research of hydraulic excavator. To control an excavator's manipulator, firstly, the Full Kinematic and dynamic model of the excavator's manipulator which three degrees of freedom were studied in [1]. The trajectory of the bucket is designed and the desired angle joint of each links are determined by using Inverse Kinematic. The controller is designed to control the bucket tracking the desired trajectory. In this paper, the computed torque control and PID control are designed. Some simulations are proposed to verify the controller.

\section{MODELING OF THE EXCAVATOR'S MAINIPULATIOR}

\subsection{Kinematic}

The kinematic and dynamic models of the experimental D Company solar 105 hydraulic excavator will be presented. These models have been built to describe the experimental excavator as a robotic manipulator with 3 degrees of freedom. In computer-controlled motion, the bucket of the excavator follows a path specified by its position and angle, i.e. the pose of the bucket, which corresponds to specific values of angular positions of the joint shafts. The values of these joint variables are determined by the lengths of hydraulic actuators. The mathematical relations between these variables are given by the kinematic relations of the excavator arm. 


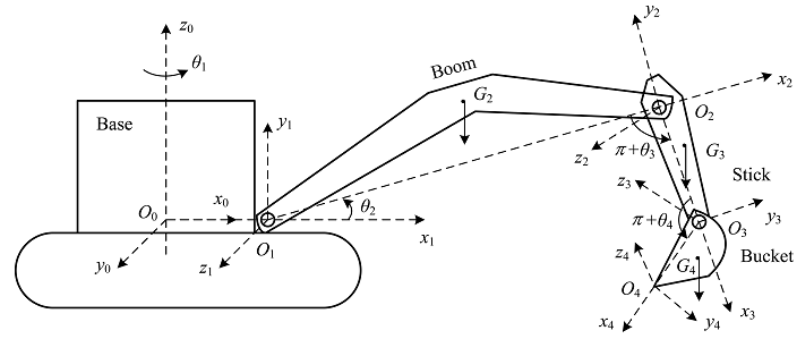

Fig. 1 Schematic diagram of an excavator

The forward kinematics is used to describe the positions and orientations of the points on the excavator in the Cartesian coordinate for the given joint positions during the digging operation. The problem can be summarized as below:

For the given $\theta=\left[\begin{array}{llll}\theta_{1} & \theta_{2} & \theta_{3} & \theta_{4}\end{array}\right]$, find the coordinate

$P=\left[\begin{array}{lll}X & Y & Z\end{array}\right]^{T}=\left[f_{x}(\theta) f_{y}(\theta) f_{z}(\theta)\right]^{T}$

To determine the positions of the points on the excavator in the base Cartesian coordinate frame, the relations between the fixed coordinate system and other coordinate systems is necessary. Therefore, the transformation matrix relating two adjacent coordinate frames was studied by Koivo [2] as follows:

$A_{i-1}^{i}=\left[\begin{array}{cccc}\cos \theta_{i} & -\cos \alpha_{i} \sin \theta_{i} & \sin \alpha_{i} \sin \theta_{i} & a_{i} \cos \theta_{i} \\ \sin \theta_{i} & \cos \alpha_{i} \cos \theta_{i} & -\sin \alpha_{i} \cos \theta_{i} & a_{i} \sin \theta_{i} \\ 0 & \sin \theta_{i} & \cos \theta_{i} & d_{i} \\ 0 & 0 & 0 & 1\end{array}\right]_{(1)}$

Where $\alpha_{i}$ is the twist angle of link $I, \mathrm{a}_{\mathrm{i}}$ is the length of link $I$, and $\mathrm{d}_{\mathrm{i}}$ is the offset distance in link $I, I=1$, $2,3,4$.

By the given coordinates of the origin in each coordinate frame $O_{i}$, the coordinates of points $O_{i}$ in the base coordinate frame can be described as follows using the equation (1):

$P_{0}^{O_{i}}=A_{0}^{i} P_{i}^{O_{i}}$

Where $P_{0}^{O_{i}}=\left[\begin{array}{lll}0 & 0 & 0\end{array}\right]^{T}$ specifies point $O_{i}$ in the ith coordinate frame. From equation (2) we can describe the origin of each coordinate frame $O_{i}$ in the base coordinate frame as follows:

$P_{0}^{O_{1}}=A_{0}^{1} P_{1}^{O_{1}}=\left[\begin{array}{llll}a_{1} c_{1} & a_{1} s_{1} & 0 & 1\end{array}\right]^{T}$

$$
\begin{aligned}
& P_{0}^{O_{2}}=A_{0}^{1} A_{1}^{2} P_{2}^{O_{2}}=\left[\begin{array}{llll}
a_{2} c_{1} c_{2}+a_{1} c_{1} & a_{2} c_{2} s_{1}+a_{1} s_{1} & a_{2} s_{2} & 1
\end{array}\right]^{T} \\
& P_{0}^{O_{3}}=A_{0}^{1} A_{1}^{2} A_{2}^{3} P_{3}^{O_{3}}=\left[\begin{array}{c}
c_{1}\left(a_{3} c_{23}+a_{2} c_{2}+a_{1}\right) \\
s_{1}\left(a_{3} c_{23}+a_{2} c_{2}+a_{1}\right) \\
a_{3} s_{23}+a_{2} s_{2} \\
1
\end{array}\right] \\
& P_{0}^{O_{4}}=A_{0}^{1} A_{1}^{2} A_{2}^{3} A_{3}^{4} P_{4} O_{4}=\left[\begin{array}{c}
c_{1}\left(a_{4} c_{234}+a_{3} c_{23}+a_{2} c_{2}+a_{1}\right) \\
s_{1}\left(a_{4} c_{234}+a_{3} c_{23}+a_{2} c_{2}+a_{1}\right) \\
a_{3} s_{23}+a_{3} s_{23}+a_{2} s_{2} \\
1
\end{array}\right]
\end{aligned}
$$

Where $\mathrm{c}_{\mathrm{i}}=\cos \theta_{\mathrm{I}}, \quad \mathrm{s}_{\mathrm{i}}=\sin \theta_{\mathrm{I}}, \quad \theta_{23}=\theta_{2}+\theta_{3}$, $\theta_{234}=\theta_{2}+\theta_{3}+\theta_{4}$.

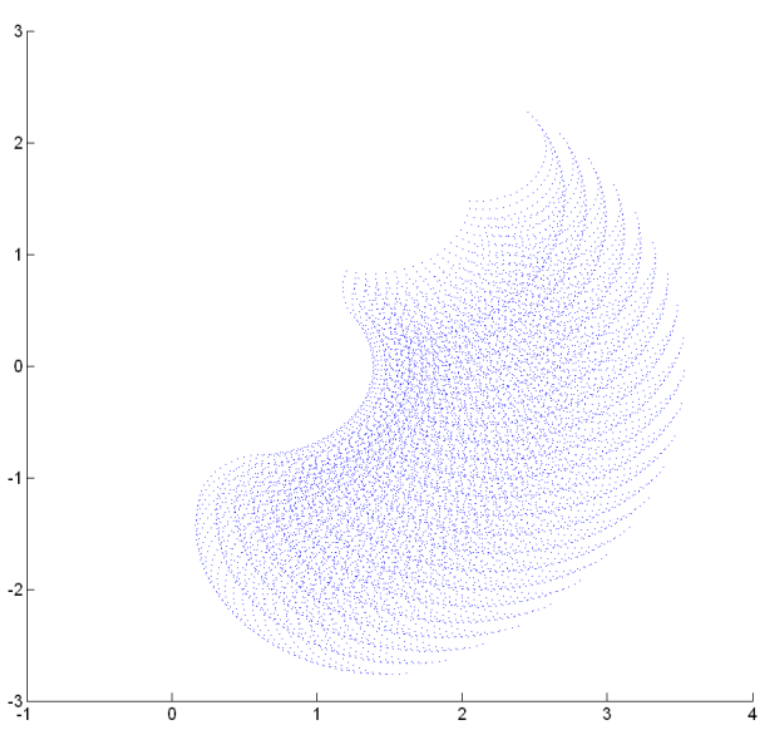

Fig. 2 The mainipulator's workspace of the Solar 105

\subsection{Inverse kinematics}

The inverse kinematic equations are used to determine the joint angles and the actuator lengths corresponding to a specific position and orientation of the bucket, given in the base coordinate system. 


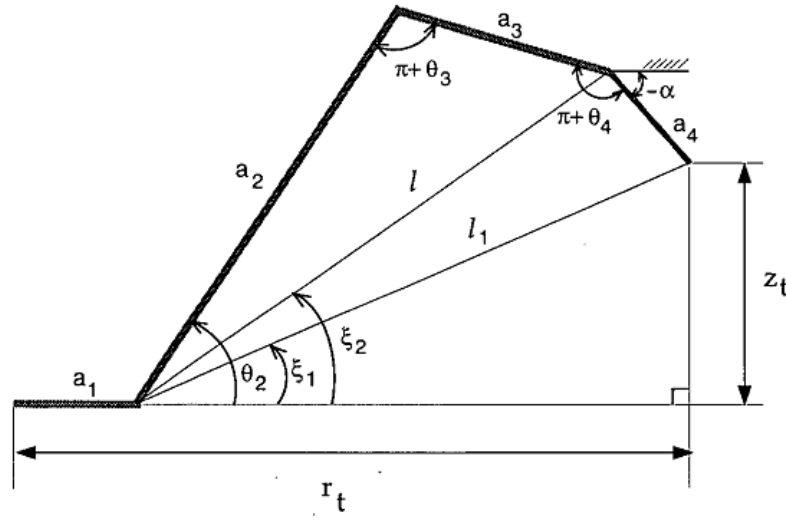

Fig. 3 Projection of the links onto the vertical plane

It is assumed that the coordinate of point $\mathrm{O}_{4} \equiv \mathrm{N}$ are given in the coordinate system. According to Tafazoli [3], the inverse kinematic model of the excavator is given as follows:

$\theta_{1}=\theta_{b}$

$l_{1}=\sqrt{\left(r_{b}-a_{1}\right)^{2}+z_{b}^{2}}$

$\xi_{1}=a \tan \left[z_{b} /\left(r_{b}-a_{1}\right)\right]$

$l=\sqrt{l_{1}^{2}+a_{4}^{2}-2 l a_{4} \cos \left(\xi_{1}-\alpha\right)}$

$\xi_{2}=a \sin \left[\left(z_{b}-a_{4} \sin \alpha_{4}\right) / l\right]$

$\theta_{2}=\xi_{2}+a \cos \left[\left(a_{2}^{2}+l^{2}-a_{3}^{2}\right) /\left(2 a_{2} l\right)\right]$

$\theta_{3}=-\pi+a \cos \left[\left(a_{2}^{2}+a_{3}^{2}-l^{2}\right) /\left(2 a_{2} a_{3}\right)\right]$

$\theta_{4}=\alpha-\theta_{23}$

\subsection{Modeling of the Excavator's manipulator}

The Dynamic model of excavator can be expressed concisely using the form of the well-known rigid-link manipulator equations of motion:

$$
D_{\alpha}(\theta) \ddot{\theta}+C_{\alpha}(\theta, \dot{\theta})+G_{\alpha}(\theta)+B_{\alpha}(\dot{\theta})=\Gamma \tau_{\alpha}-F_{L}
$$

Where

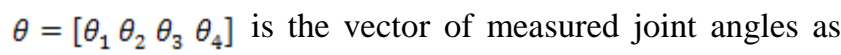
defined in Fig.2

$D_{\alpha}(\theta)$ represents inertia

$c_{\alpha}(\theta, \dot{\theta})$ represents Coriolis and centripetal effects

$G_{\alpha}(\theta)$ represents gravity forces

$B_{\alpha}(\dot{\theta})$ represents frictions

$\Gamma$ is the corresponding input matrix

$\tau_{\alpha}=\left[\begin{array}{llll}\tau_{1} & \tau_{2} & \tau_{3} & \tau_{4}\end{array}\right]^{T}$ specifies the torques acting on the joint shafts

$F_{L}$ represents the interactive torques between the bucket and the environment during the digging operation.

\section{CONTROL OF EXCAVATOR}

The control system shown in figure 4 is designed to make the end of effecter track desired trajectory. Using the dynamic model of excavator in (15), the primary controller is given below:

$$
U_{d}=\hat{D}(\theta) \ddot{\theta}_{v}+\hat{C}(\theta, \dot{\theta}) \dot{\theta}+\hat{G}(\theta)+\hat{B}(\dot{\theta})
$$

Where

$\dot{\theta}_{v}=\ddot{\theta}_{d}-k_{v} e-k_{p} e ; e=\theta-\theta_{d}$ is the error $\mathrm{k}_{\mathrm{v}}$ and $\mathrm{k}_{\mathrm{p}}$ are the linear gains to be determined.

$\hat{D}(\theta)$ is the estimated inertia, $\hat{C}(\theta, \dot{\theta})$ is the estimated

Coriolis and centripetal effects, $\hat{G}(\theta)$ is the estimated gravity forces; $\hat{B}(\dot{\theta})$ is the estimated friction effects; $\mathrm{U}_{\mathrm{d}}$ is the desired torque provided to the system; $\theta_{d}, \dot{\theta}_{d}, \ddot{\theta}_{d}$ are the desired joint link angle, angular velocity and angular acceleration.

A secondary controller is designed to compensate for derivations of the actual motion from the desired 
trajectory [2].

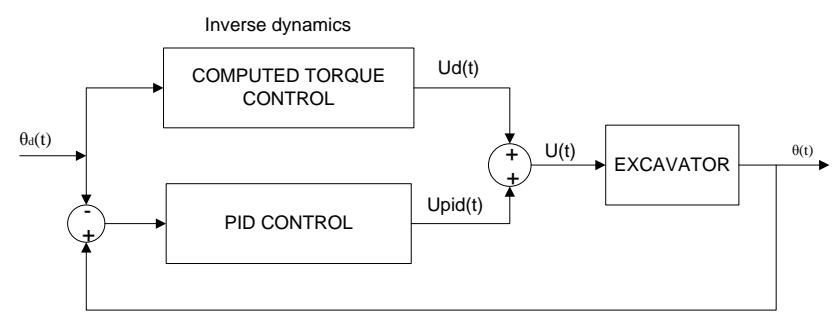

Fig.4 Control architecture

\section{SIMULATION}

A simulation study on an excavator was performed in Matlab/Simulink. A desired trajectory is first designed in the Casterian space. The joint angles of the boom, arm and bucket are determined by using Inverse Kinematic.

In figure 5 shows the result of the simulation when the trajectory of the bucket is moving horizontal.

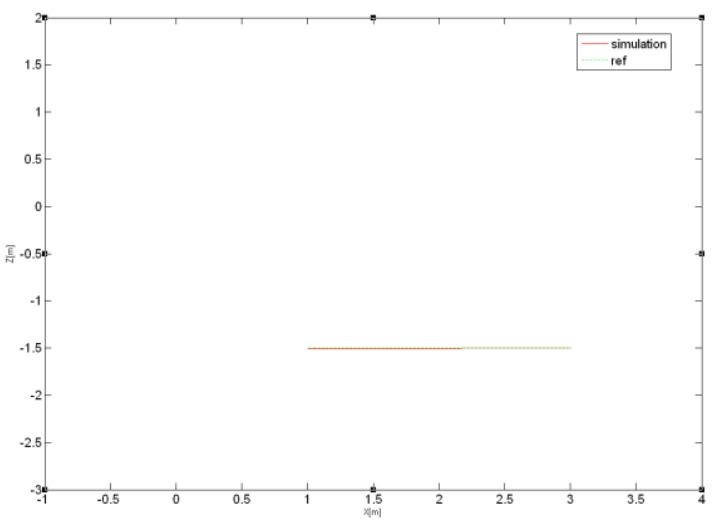

(a)

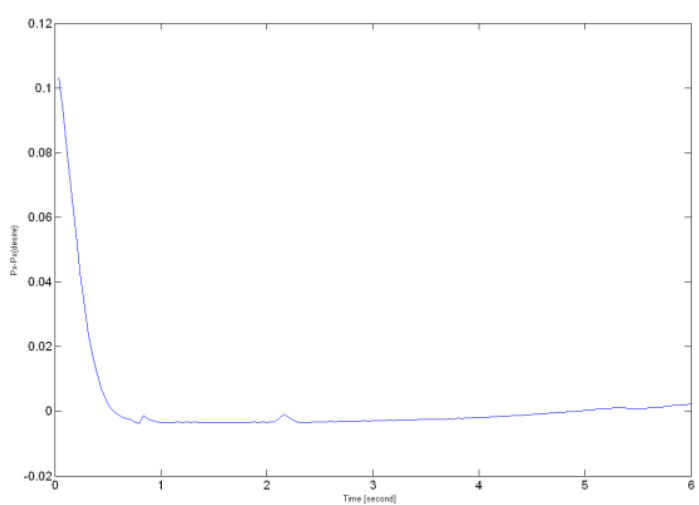

(b)

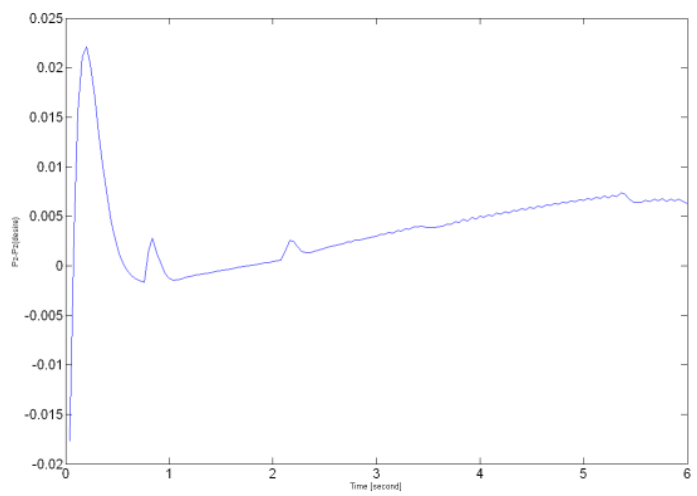

(c)

Fig.5 Horizontal movement (m): (a) Trajectory of the bucket; (b) x-coordinate Error of bucket Position versus time; (c) z-coordinate Error of bucket Position versus time.

The error of the angular joint of boom, arm and bucket are shown in the figure 6 . The errors of all the links of system when the bucket is moving are less than 1 degree.

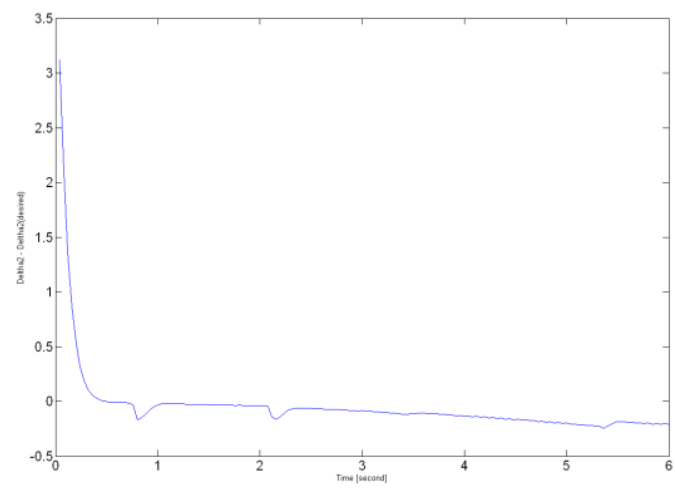

(a)

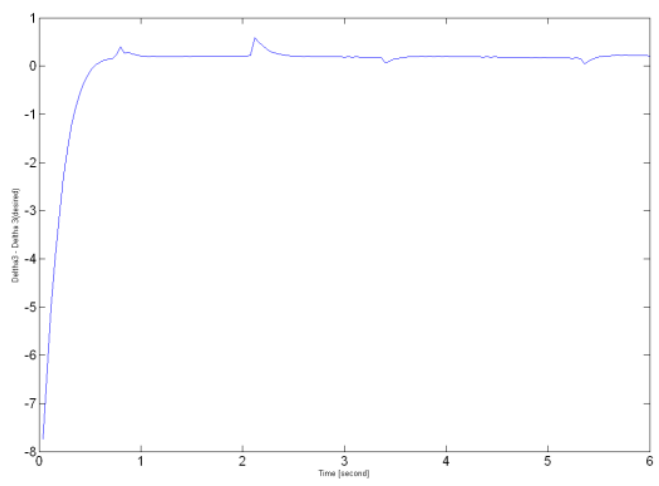

(b) 


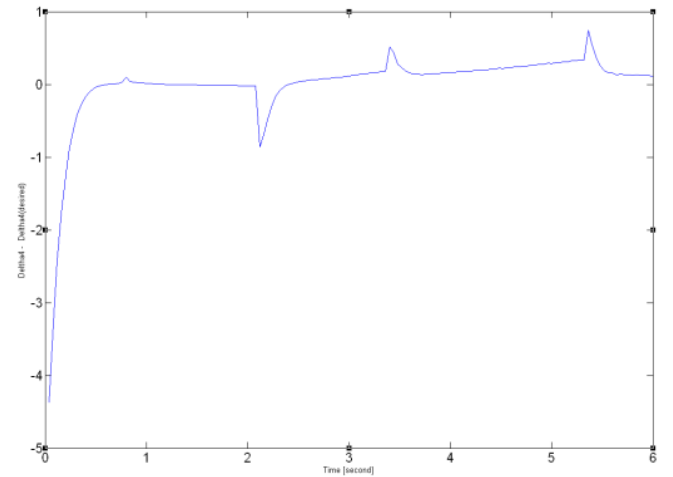

(c)

Fig. 6 The error of the angular joint

(a) Boom; (b) Arm; (c) Bucket (degree)

Another simulation is shown in the figure 7 when the end of effecter is moving vertical.

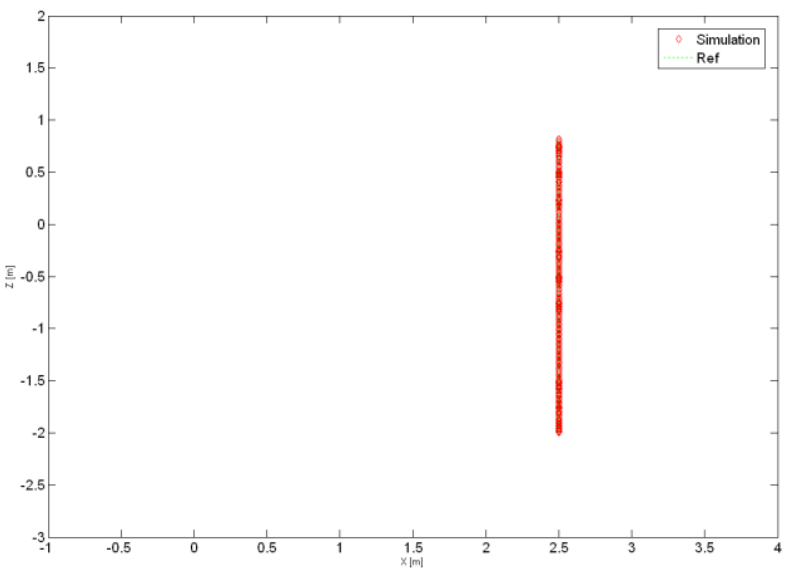

(a)

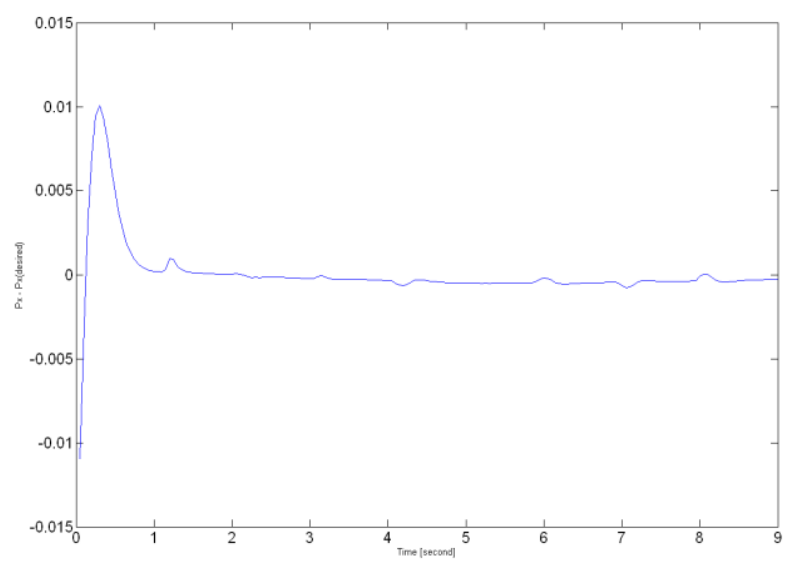

(b)

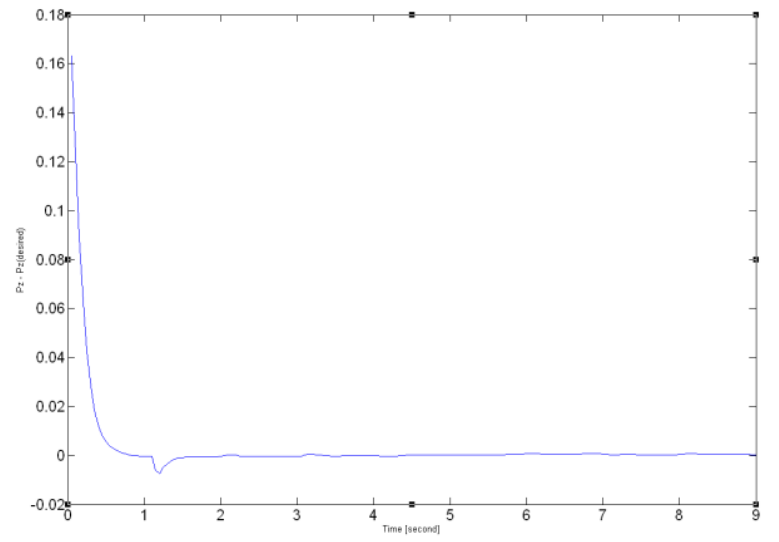

(c)

Fig.7 Vertical movement (m): (a) Trajectory of the bucket; (b) X-coordinate Error of bucket Position versus time; (c) z-coordinate Error of bucket Position versus time.

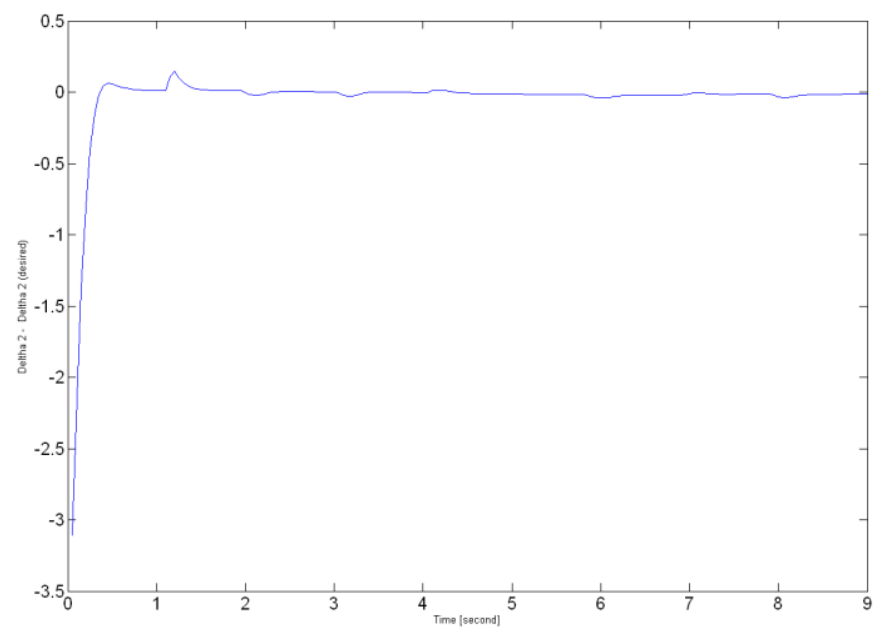

(a)

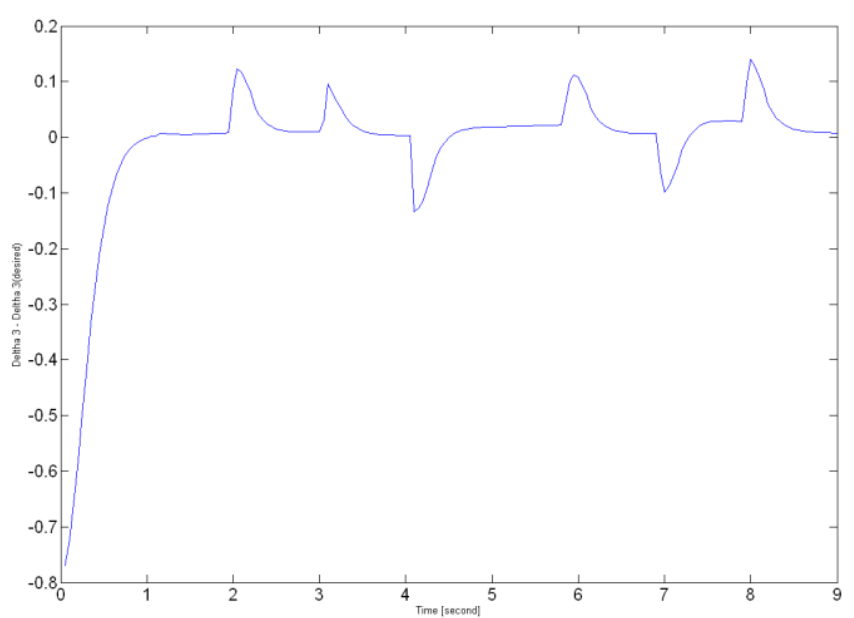

(b) 


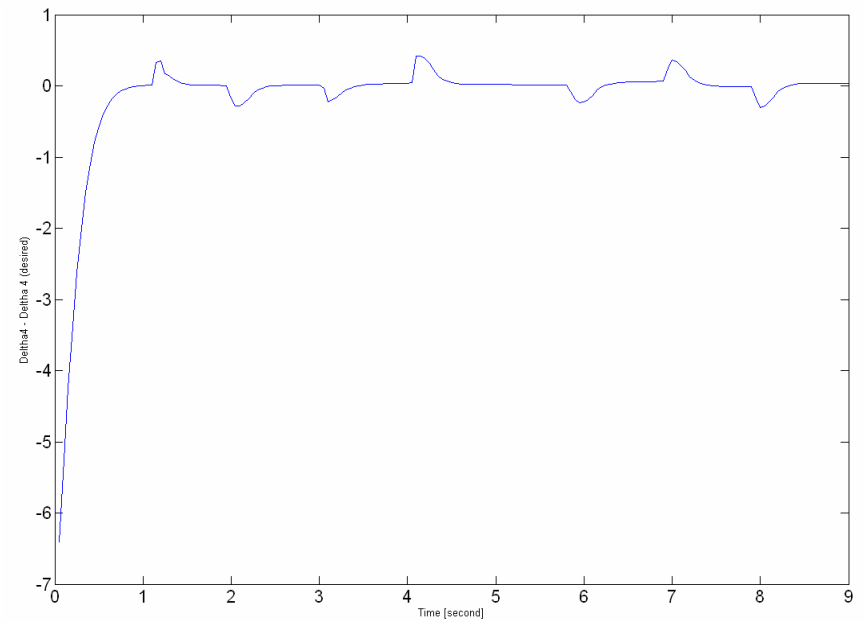

(c)

Fig. 8 The error of the angular joint (a) Boom; (b) Arm; (c) Bucket (degree)

The error of the angular joint of the boom, arm and bucket when the trajectory of the end of effecter is moving vertical is shown in figure 8 . The errors of all the links of system when the bucket is moving are less than 0.5 degree. The simulations illustrate that the controller performs very well during the system is operating.

\section{CONCLUSION}

The paper has reported the review of the Kinematic and modeling of the Excavator's Manipulator. The basic controller is computed torque control and PID control are applied to the system and some simulations are proposed to test the controller.

\section{References}

[1] Koivo, A.J. (1994) 'Kinematics of excavators (backhoes) for transferring surface material', J. Aerosp. Eng., Vol. 7, No. 1, pp.17-32.

[2] Koivo, A.J., Thoma, M., Kocaoglan, E. and AndradeCetto, J. (1996) 'Modeling and control of excavator dynamics during digging operation', J. Aerosp. Eng., January, Vol. 9, No. 1, pp.10-18.

[3] Tafazoli. S, P. D. Lawrence, S. E. Salcudean. 'Identification of inertial and friction parameters for excavator arms'. IEEE Transactions on Robotics and Automation, vol. 15, no.5, pp.996-971, 1999.
[4]Tafazoli, S., Salcudean, S.E., Hashtrudi-Zaad, K. and Lawrence, P.D. (2002) 'Impedance control of a teleoperated excavator', IEEE Trans. on Control Systems Technology, May, Vol. 10, No. 3, pp.355-367.

[5] Hongnian Yu*, Yang Liu and Mohammad Shahidul Hasan, 'Review of modelling and remote control for Excavators', Int. J. Advanced Mechatronic Systems, Vol. 2, Nos. 1/2, 2010.

[6] Yang Liu, Mohammad Shahidul Hassan, Hong-Nian Yu, Modeling and remote control of an Excavator', International Journal of Automation and Computing, 7(3), August 2010, 349-358.

[7] Ha, Q., Santos, M., Nguyen, Q., Rye, D. and DurrantWhyte, H. (2002) 'Robotic excavation in construction automation', IEEE Robotics \& Automation Magazine, March, Vol. 9, No. 1, pp.20-28.

[8] Ha, Q.P., Nguyen, Q.H., Rye, D.C. and Durrant-Whyte, H.F. (2000) 'Impedance control of a hydraulically-actuated robotic excavator', Automat. Construction, Vol. 9, No. 5, pp.421-435.

[9] ZHANG Da-qing, HE Qing-hua, HAO Peng. Trajectory tracking control of hydraulic excavator bucket [J]. Journal of Jilin University: Engineering and Technology Edition, 2005, 35(5): 490-494. 